\title{
A|P| Journal of
}

\section{Nonlinear modulation of TM waves in a circular waveguide}

Omar R. Asfar and Ali H. Nayfeh

Citation: Journal of Applied Physics 47, 88 (1976); doi: 10.1063/1.322302

View online: http://dx.doi.org/10.1063/1.322302

View Table of Contents: http://scitation.aip.org/content/aip/journal/jap/47/1?ver=pdfcov

Published by the AIP Publishing

\section{Articles you may be interested in}

Nonlinear polymer-clad silicon slot waveguide modulator with a half wave voltage of $0.25 \mathrm{~V}$

Appl. Phys. Lett. 92, 163303 (2008); 10.1063/1.2909656

Linear theory of the electron cyclotron maser based on TM circular waveguide mode

Phys. Plasmas 13, 073104 (2006); 10.1063/1.2221678

Control of TM waves by strong TE nonlinear guided waves

Appl. Phys. Lett. 52, 1 (1988); 10.1063/1.99326

Wave Propagation in a Circular Waveguide with an Absorbing Wall

J. Appl. Phys. 41, 433 (1970); 10.1063/1.1658369

The Susceptance of a Thin Iris in Circular Wave Guide with the TM 01 Mode Incident J. Appl. Phys. 22, 1316 (1951); 10.1063/1.1699857

MIT LINCOLN LABORATORY CAREERS

Discover the satisfaction of innovation and service to the nation
- Space Control

- Air \& Missile Defense

- Communications Systems \& Cyber Security

- Intelligence, Surveillance and Reconnaissance Systems

- Advanced
Electronics
- Tactical Systems
" Homeland
Protection
- Air Traffic Control

- Air Traffic Control

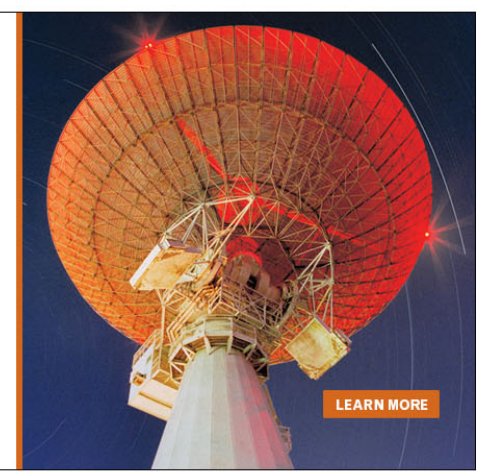




\title{
Nonlinear modulation of TM waves in a circular waveguide
}

\author{
Omar R. Asfar* and Ali H. Nayfeh \\ Department of Engineering Science and Mechanics, Virginia Polytechnic Institute and State University, \\ Blacksburg, Virginia 24061 \\ (Received 6 February 1975; in final form 23 September 1975)

\begin{abstract}
The method of multiple scales is used to derive a nonlinear Schrödinger equation for the temporal and spatial amplitude and phase modulations of TM waves in a perfectly conducting guide containing a nonlinear isotropic medium. This equation is used to show that monochromatic waves are stable if the mechanism producing the nonlinearity is an electric or magnetic polarization and unstable if the nonlinearity is due to electrostriction or magnetostriction. It is also used to determine the amplitude dependence of the cutoff frequencies.
\end{abstract}

PACS numbers: $84.40 . \mathrm{S}$

\section{INTRODUCTION}

Electromagnetic wave propagation in a waveguide containing a nonlinear isotropic medium was treated by Keller and Millman ${ }^{1}$ using a generalization of the Lindstedt-Poincaré perturbation technique; they presented numerical results for rectangular waveguides only. Taniuti and Yajima ${ }^{2}$ used the method of multiple scales in order to derive a nonlinear Schrödinger equation for nonlinear wave modulation and applied the technique to nonlinear optics. ${ }^{3}$

In this paper, we derive a nonlinear Schrödinger equation governing amplitude and phase modulations in a circular waveguide using the method of multiple scales. ${ }^{4}$ We follow Nayfeh's treatment of the propagation of a wave packet in a hard-walled circular acoustic duct ${ }^{5}$ and use Schrödinger's equation to study the stability of monochromatic wave solutions. The mechanisms producing the nonlinearity play a key role in deciding the stability of monochromatic wave solutions in much the same way that it characterizes self-focusing and defocusing of laser beams ${ }^{6}$ in nonlinear optics.

\section{FORMULATION}

We consider a circular waveguide for long-distance transmission of microwave power filled with a homogeneous isotropic nonlinear medium whose constitutive relations are given by

$$
\mathrm{D}=\epsilon(|\mathrm{E}|) \mathrm{E}, \quad \mathrm{B}=\mu(|\mathrm{H}|) \mathrm{H} .
$$

The dielectric constant and the magnetic permeability are assumed to have the following isotropic nonlinear functions:

$$
\begin{aligned}
& \epsilon=\epsilon_{0}\left(1+\epsilon_{2}|\mathbf{E}|^{2}+\ldots\right), \\
& \mu=\mu_{0}\left(1+\mu_{2}|\mathbf{H}|^{2}+\cdots\right),
\end{aligned}
$$

where $\epsilon_{0}$ and $\mu_{0}$ correspond to the linear problem while $\epsilon_{2}$ and $\mu_{2}$ are the nonlinear indices. We use the cylindrical coordinates $\rho^{\prime}, \phi, z^{\prime}$ and introduce dimensionless variables $\rho$ and $z$ by using the radius of the cylinder $R$ as a reference quantity so that $\rho=\rho^{\prime} / R$ and $z=z^{\prime} / R$. We also make time dimensionless by using $R$ and the linear phase speed of the waves in the medium $v$ $=\left(\mu_{0} \epsilon_{0}\right)^{-1 / 2}$ so that $t=v t^{\prime} / R$ is the dimensionless time.

For nonlinear media, the Maxwell equations can be reduced to the following governing equation:

$$
\begin{aligned}
\nabla^{2} \mathbf{E} & +\nabla\left(\frac{1}{\epsilon}(\mathbf{E} \cdot \nabla \epsilon)\right) \\
= & \left(\mu_{0} \epsilon_{0}\right)^{-1 / 2} \frac{\partial}{\partial t}\left(\left(\mu_{0} \epsilon_{0}\right)^{-1 / 2} \mu \frac{\partial}{\partial t}(\epsilon \mathbf{E})+\nabla \mu \times \mathbf{H}\right) .
\end{aligned}
$$

The boundary condition to be satisfied in the case of $\mathrm{TM}$ waves and perfectly conducting walls is

$$
E_{z}=0 \text { at } \rho=1 \text {. }
$$

To determine an approximate solution for $\mathrm{Eq}$. (3) subject to boundary condition (4), for small but finite amplitudes, we use the method of multiple scales and let

$$
\begin{aligned}
& \mathrm{E}(\rho, \phi, z, t)=\sum_{n=1}^{3} \delta^{n} \mathrm{E}_{n}\left(\rho, \phi, Z_{0}, Z_{1}, Z_{2}, T_{0}, T_{1}, T_{2}\right)+0\left(\delta^{4}\right), \\
& \mathrm{H}(\rho, \phi, z, t)=\sum_{n=1}^{3} \delta^{n} \mathrm{H}_{n}\left(\rho, \phi, Z_{0}, Z_{1}, Z_{2}, T_{0}, T_{1}, T_{2}\right)+0\left(\delta^{4}\right),
\end{aligned}
$$

where $\delta$ is a small but finite dimensionless parameter characterizing the amplitude of the wave and

$$
Z_{m}=\delta^{m} z, \quad T_{m}=\delta^{m} t .
$$

Here $Z_{0}$ is a length scale of the order of a wavelength, $Z_{1}$ and $Z_{2}$ are length scales characterizing the slow spatial amplitude and phase modulations, $T_{0}$ is a time scale of the order of a period of the wave, and $T_{1}$ and $T_{2}$ are time scales characterizing the slow temporal amplitude and phase modulations. Using Eq. (6) and the chain rule, we express the temporal and axial derivatives as

$$
\begin{aligned}
& \frac{\partial}{\partial z}=\frac{\partial}{\partial Z_{0}}+\delta \frac{\partial}{\partial Z_{1}}+\delta^{2} \frac{\partial}{\partial Z_{2}}+\ldots, \\
& \frac{\partial}{\partial t}=\frac{\partial}{\partial T_{0}}+\delta \frac{\partial}{\partial T_{1}}+\delta^{2} \frac{\partial}{\partial T_{2}}+\cdots .
\end{aligned}
$$

Substituting Eqs. (5)-(7) into Eqs. (3) and (4), using Eq. (2) and equating coefficients of like powers of $\delta$, we obtain the following for $E_{8}$ :

Order $\delta$

$$
\begin{aligned}
& L\left(E_{1 \varepsilon}\right) \equiv \nabla_{0}^{2} E_{1 z}-\frac{\partial^{2} E_{1 z}}{\partial T_{0}^{2}}=0, \\
& \nabla_{0}^{2}=\frac{\partial^{2}}{\partial \rho^{2}}+\frac{1}{\rho} \frac{\partial}{\partial \rho}+\frac{1}{\rho^{2}} \frac{\partial^{2}}{\partial \phi^{2}}+\frac{\partial^{2}}{\partial Z_{0}^{2}},
\end{aligned}
$$




$$
E_{1 z}=0 \text { at } \rho=1
$$

Order $\delta^{2}$

$$
\begin{aligned}
& L\left(E_{2 z}\right)=2\left(\frac{\partial^{2} E_{1 \varepsilon}}{\partial T_{0} \partial T_{1}}-\frac{\partial^{2} E_{1 \varepsilon}}{\partial Z_{0} \partial Z_{1}}\right), \\
& E_{2 z}=0 \quad \text { at } \rho=1 .
\end{aligned}
$$

Order $\delta^{3}$

$$
\begin{aligned}
L\left(E_{3 s}\right) & \\
= & \frac{\partial^{2} E_{1 z}}{\partial T_{1}^{2}}+2 \frac{\partial^{2} E_{1 z}}{\partial T_{0} \partial T_{2}}+2 \frac{\partial^{2} E_{2 z}}{\partial T_{0} \partial T_{1}}-\frac{\partial^{2} E_{1 z}}{\partial Z_{1}^{2}}-2 \frac{\partial^{2} E_{1 z}}{\partial Z_{0} \partial Z_{2}} \\
& -2 \frac{\partial^{2} E_{2 z}}{\partial Z_{0} \partial Z_{1}}+\epsilon_{2} \frac{\partial^{2}}{\partial T_{0}^{2}}\left(\left|E_{1}\right|^{2} E_{1 z}\right)+\mu_{2} \frac{\partial}{\partial T_{0}}\left(\left|\mathrm{H}_{1}\right|^{2} \frac{\partial E_{1 z}}{\partial T_{0}}\right) \\
& +\mu_{2}\left(\frac{\mu_{0}}{\epsilon_{0}}\right)^{1 / 2} \frac{\partial}{\partial T_{0}}\left(\nabla_{0}\left(\left|\mathrm{H}_{1}\right|^{2}\right) \times \mathrm{H}_{1}\right)_{z} \\
& -\epsilon_{2} \frac{\partial}{\partial Z_{0}}\left(\nabla_{0}\left(\left|\mathrm{E}_{1}\right|^{2}\right) \cdot \mathrm{E}_{1}\right), \\
\nabla_{0}= & \hat{e}_{\rho} \frac{\partial}{\partial \rho}+\hat{e}_{\phi} \frac{\partial}{\partial \phi}+\hat{e}_{z} \frac{\partial}{\partial Z_{0}}, \\
E_{3 z}= & 0 \quad \text { at } \rho=1 .
\end{aligned}
$$

Here, $\hat{e}_{\rho}, \hat{e}_{\phi}$, and $\hat{e}_{s}$ are unit vectors in the $\rho, \phi$, and $z$ directions, respectively.

\section{SOLUTION}

We take the solution of Eq. (8) that is bounded at the axis in the form of a traveling wave packet centered at frequency $\omega$ and wavenumber $k_{s}$; that is, we let

$$
\begin{aligned}
& E_{1 \varepsilon}=A\left(Z_{1}, Z_{2}, T_{1}, T_{2}\right) J_{m}\left(k_{\rho} \rho\right) \exp (i \theta)+\mathrm{cc}, \\
& \theta=k_{z} Z_{0}-\omega T_{0}+m \phi,
\end{aligned}
$$

where $J_{m}$ is Bessel's function of order $m$, cc represents the complex conjugate of the preceding terms, and $\omega$ and $k_{z}$ satisfy the dispersion relationship

$$
\omega^{2}-k_{\varepsilon}^{2}=k_{\rho}^{2} \text {. }
$$

Substituting Eq. (11) into Eq. (8c), we have

$$
J_{m}\left(k_{p}\right)=0 \text {. }
$$

To carry the solution to higher orders, we have to determine the remaining field components in terms of $E_{1 z}$. Manipulation of the Faraday and A mpère laws in the set of Maxwell equations and use of the explicit $Z_{0}$ dependence [Eq. (11)] leads to the determination of the transverse fields as

$$
\begin{aligned}
& E_{1 \rho}=i\left(k_{z} / k_{\rho}\right) A J_{m}^{\prime}\left(k_{\rho} \rho\right) \exp (i \theta)+\mathrm{cc}, \\
& E_{1 \Phi}=-(m / \rho)\left(k_{z} / k_{\rho}^{2}\right) A J_{m}\left(k_{\rho} \rho\right) \exp (i \theta)+c c, \\
& \left.H_{1 \rho}=\left(m \omega / \rho k_{\rho}^{2}\right) \epsilon_{0} / \mu_{0}\right)^{1 / 2} A J_{m}\left(k_{\rho} \rho\right) \exp (i \theta)+c c, \\
& H_{1 \Phi}=i\left(\omega / k_{\rho}\right)\left(\epsilon_{0} / \mu_{0}\right)^{1 / 2} A J_{m}^{\prime}\left(k_{\rho} \rho\right) \exp (i \theta)+\mathrm{cc} .
\end{aligned}
$$

The function $A$ is not determined at this level of approximation; it will be determined from the solvability conditions of the second- and third-order problems.

Substituting Eq. (11) into Eq. (9), we obtain

$$
L\left(E_{2 \varepsilon}\right)=-2 i\left(\omega \frac{\partial A}{\partial T_{1}}+k_{z} \frac{\partial A}{\partial Z_{1}}\right) J_{m}\left(k_{\rho} \rho\right) \exp (i \theta)+c c .
$$

Secular terms will be eliminated if we let

$$
\omega \frac{\partial A}{\partial T_{1}}+k_{\varepsilon} \frac{\partial A}{\partial Z_{1}}=0 .
$$

Since the homogeneous solution of Eq. (15) is proportional to $E_{1 z}$, one can set it equal zero, without loss of generality. If it were included, the results will not change provided that one redefines the amplitude and the phase, though the algebra becomes more involved. Thus, in this paper, we choose

$$
\mathbf{E}_{2}=0 \text {. }
$$

Substituting Eqs. (11), (14), and (17) into Eq. (10a) and carrying out the indicated algebraic manipulations, we obtain

$$
\begin{aligned}
L\left(E_{3 \varepsilon}\right)= & {\left[-2 i\left(\omega \frac{\partial A}{\partial T_{2}}+k_{\ell} \frac{\partial A}{\partial Z_{2}}\right)+\frac{\partial^{2} A}{\partial T_{2}^{2}}-\frac{\partial^{2} A}{\partial Z_{1}^{2}}\right] } \\
& \times J_{m}\left(k_{\rho} \rho\right) \exp (i \theta)+A^{2} \bar{A} F(\rho) \exp (i \theta)+\mathrm{NST}+\mathrm{cc},
\end{aligned}
$$

where $F(\rho)$ is given in the Appendix and NST stands for terms that do not produce secular terms, $i_{\text {. e. }}$, terms involving higher harmonics in $\theta$. Since the homogeneous third-order problem consisting of Eqs. (10c) and (18) has a nontrivial solution, the corresponding inhomogeneous problem has a solution if and only if a solvability condition is satisfied.

To determine this solvability condition, we seek a particular solution of the form

$$
E_{3 \varepsilon}=\psi\left(\rho, Z_{1}, Z_{2}, T_{1}, T_{2}\right) \exp (i \theta)+\mathrm{cc} .
$$

Substituting this solution into Eqs. (10c) and (18) and equating the coefficients of $\exp (i \theta)$ on both sides, we obtain

$$
\begin{aligned}
& \frac{\partial^{2} \psi}{\partial \rho^{2}}+\frac{1}{\rho} \frac{\partial \psi}{\partial \rho}+\left(k_{\rho}^{2}-\frac{m^{2}}{\rho^{2}}\right) \psi \\
&=-\left[2 i\left(\omega \frac{\partial A}{\partial T_{\mathrm{a}}}+k_{z} \frac{\partial A}{\partial Z_{2}}\right)-\frac{\partial^{2} A}{\partial T_{1}^{2}}+\frac{\partial^{2} A}{\partial Z_{1}^{2}}\right] J_{m}\left(k_{o} \rho\right) \\
&+A^{2} \bar{A} F(\rho), \\
& \psi=0 \quad \text { at } \rho=1 .
\end{aligned}
$$

Multiplying Eq. $(20)$ by $\rho J_{m}\left(k_{\rho} \rho\right)$, integrating by parts from $\rho=0$ to $\rho=1$, and using Eq. (21), we obtain the solvability condition

$$
2 i\left(\omega \frac{\partial A}{\partial T_{2}}+k_{z} \frac{\partial A}{\partial Z_{2}}\right)-\frac{\partial^{2} A}{\partial T_{1}^{2}}+\frac{\partial^{2} A}{\partial Z_{1}^{2}}=\Lambda A^{2} \bar{A},
$$

where

$$
\Lambda=\left[\int_{0}^{1} \rho F(\rho) J_{m}\left(k_{\rho} \rho\right) d \rho\right]\left[\int_{0}^{1} \rho J_{m}^{2}\left(k_{\rho} \rho\right) d \rho\right]^{-1} .
$$

Eliminating $\partial^{2} A / \partial Z_{1}^{2}$ from Eq. (22) by using Eq. (16), we obtain

$$
2 i\left(\omega \frac{\partial A}{\partial T_{2}}+k_{z} \frac{\partial A}{\partial Z_{2}}\right)+\left(\frac{\omega^{2}}{k_{z}^{2}}-1\right) \frac{\partial^{2} A}{\partial T_{1}^{2}}=\Lambda A^{2} \bar{A} .
$$

To simplify Eq. (24), we differentiate the dispersion relationship [Eq. (12)] with respect to $\omega$ and obtain

$$
k_{z} k_{\varepsilon}^{\prime}=\omega,
$$


where $k_{z}^{\prime}=d k_{g} / d \omega$, the inverse of the group velocity. Differentiating Eq. (25) with respect to $\omega$, we obtain

$$
k_{z} k_{z}^{\prime \prime}=1-k_{z}^{\prime 2}=1-\omega^{2} / k_{z}^{2} .
$$

Using Eqs. (25) and (26), letting $T_{n}=\delta^{n} t$ and $Z_{n}=\delta^{n} z$, and rearranging terms, we rewrite $\mathrm{Eq}$. (24) as

$$
\frac{\partial A}{\partial z}+k_{z}^{\prime} \frac{\partial A}{\partial t}+\frac{1}{2} i k_{z}^{\prime \prime} \frac{\partial^{2} A}{\partial t^{2}}=-\frac{1}{2} i \delta^{2} \frac{\Lambda}{k_{z}} A^{2} \bar{A} .
$$

Changing the independent variables from $z$ and $t$ to

$$
\xi=t-k_{z}^{\prime} z, \quad \eta=z,
$$

we express $\mathrm{Eq} .(27)$ in the form

$$
\frac{\partial A}{\partial \eta}+\frac{1}{2} i k_{\varepsilon}^{\prime \prime} \frac{\partial^{2} A}{\partial \xi^{2}}=-\frac{1}{2} i \delta^{2} \frac{\Lambda}{k_{z}} A^{2} \bar{A},
$$

which is a nonlinear Schrödinger equation. Letting $A$ $=\frac{1}{2} a \exp (i \beta)$ with real $a$ and $\beta$ in Eq. (29) and separating real and imaginary parts, we obtain

$$
\begin{aligned}
& \frac{\partial a}{\partial \eta}-k_{z}^{\prime \prime}\left(\frac{\partial a}{\partial \xi} \frac{\partial \beta}{\partial \xi}+\frac{1}{2} a \frac{\partial^{2} \beta}{\partial \xi^{2}}\right)=0, \\
& \frac{\partial \beta}{\partial \eta}+\frac{1}{2} k_{z}^{\prime \prime}\left[\frac{1}{a} \frac{\partial^{2} a}{\partial \xi^{2}}-\left(\frac{\partial \beta}{\partial \xi}\right)^{2}\right]=-\frac{1}{8} \delta^{2} \frac{\Lambda}{k_{z}} a^{2} .
\end{aligned}
$$

\section{The case of monochromatic waves}

For monochromatic waves, $\partial a / \partial \xi=\partial \beta / \partial \xi=0$, and Eqs. (30) and (31) can be integrated to give

$$
a=a_{0} \quad \text { and } \beta=-\frac{1}{8} \delta^{2}\left(\Lambda / k_{z}\right) a_{0}^{2} \eta+\beta_{0},
$$

where $a_{0}$ and $\beta_{0}$ are constants. Substituting for $a$ and $\beta$ from Eq. (32) into Eq. (11), we rewrite $E_{z}$ in the form

$$
E_{z}=\delta a_{0} J_{m}\left(k_{\rho} \rho\right) \cos \left(k z-\omega t+m \phi+\beta_{0}\right)+0\left(\delta^{3}\right),
$$

where

$$
k=k_{z}-\frac{1}{8} \delta^{2} \Lambda k_{z}^{-1} a_{0}^{2} .
$$

Equation (34) shows that the nonlinearity of the medium results in a wavenumber shift from $k_{z}$ to $k$. To determine whether this shift increases or decreases the wavenumber one has to examine the sign of the nonlinear factor $\Lambda$. This depends on the signs of $\mu_{2}$ and $\epsilon_{2}$ which, in turn, depend on the mechanism producing the nonlinearity, a point we shall consider in some detail in the following discussion on the stability of monochromatic waves.

Equations (30) and (31) can be used to analyze the stability of the monochromatic wave solution, Eqs. and (34). To do this, we let

$$
a=a_{0}+a_{1}, \quad \beta=-\frac{1}{8} \delta^{2}\left(\Lambda / k_{z}\right) a_{0}^{2} \eta+\beta_{0}+\beta_{1},
$$

where $a_{1}$ and $\beta_{1}$ are small compared with the preceding terms. Substituting Eq. (35) into Eqs. (30) and (31) and neglecting the nonlinear terms in $a_{1}$ and $\beta_{1}$, we obtain

$$
\begin{aligned}
& \frac{\partial a_{1}}{\partial \eta}-\frac{1}{2} k_{z}^{\prime \prime} a_{0} \frac{\partial^{2} \beta_{1}}{\partial \xi^{2}}=0, \\
& \frac{\partial \beta_{1}}{\partial \eta}+\frac{1}{2} k_{z}^{\prime \prime} a_{0}^{-1} \frac{\partial^{2} a_{1}}{\partial \xi^{2}}=-\frac{1}{4} \delta^{2} \frac{\Lambda}{k_{z}} a_{0} a_{1} .
\end{aligned}
$$

Since Eqs. (36) and (37) are linear, we seek their solution in the form

$$
\begin{aligned}
& a_{1}=\tilde{a}_{1} \exp [i(\tilde{k} \eta-\tilde{\omega} \xi)], \\
& \beta_{1}=\tilde{\beta}_{1} \exp [i(\tilde{k} \eta-\tilde{\omega} \xi)],
\end{aligned}
$$

where $\tilde{a}_{1}$ and $\widetilde{\beta}_{1}$ are constants. Substituting this solution into Eqs. (36) and (37) and eliminating $\widetilde{a}_{1}$ and $\widetilde{\beta}_{1}$, we obtain

$$
\tilde{k}^{2}=\frac{1}{4} k_{z}^{\prime \prime 2} \tilde{\omega}^{2}\left(\tilde{\omega}^{2}-\frac{1}{2} \delta^{2} \Lambda a_{0}^{2} / k_{z} k_{z}^{\prime \prime}\right),
$$

which shows that if $\Lambda / k_{z}^{\prime \prime}<0, \tilde{k}$ is always real for all values of $\tilde{\omega}$ so that monochromatic waves are neutrally stable. On the other hand, if $\Lambda / k_{z}^{\prime \prime}>0, \widetilde{k}^{2}$ is negative for all $\tilde{\omega}<\delta a_{0}\left(\Lambda / 2 k_{z} k_{z}^{\prime \prime}\right)^{1 / 2}$; consequently, disturbances grow exponentially with $\eta$ and monochromatic waves are unstable. Now, Eqs. (12) and (26) show that $k_{z}^{\prime \prime}=-k_{\mathrm{p}}^{2} / k_{\varepsilon}^{3}$ $<0$; hence, the stability of monochromatic waves depends on the sign of $\Lambda$ 。

Apart from the signs of $\epsilon_{2}$ and $\mu_{2}$, our unpublished numerical results show that the signs of the coefficients of $\epsilon_{2}$ and $\mu_{2}$ are always negative in the microwave region. As previously mentioned, the signs of $\epsilon_{2}$ and $\mu_{2}$ depend on the mechanism responsible for the nonlinear behavior. Considering a nonlinear dielectric medium, we have the following possibilities according to Ref. 6: (i) nonlinear polarization due to saturation of the electronic polarizability which means $\epsilon_{2}<0$, and hence $\Lambda / k_{z}^{\prime \prime}<0$ (neutral stability); (ii) electrostriction gives rise to $\epsilon_{2}>0$, and hence $\Lambda / k_{z}^{\prime \prime}>0$ (instability).

In a medium where magnetic properties are dominant the mechanisms responsible for nonlinearity are nonlinear polarization and magnetostriction. The same conclusions regarding stability in the case of dielectric media hold correspondingly in the magnetic case. We thus see how the type of material is important for the question of stability. If both strictional effects and polarization are present in a material, they will compete. Usually strictional effects dominate thereby producing instability.

\section{Solution near cutoff frequencies}

Although Eqs. (33) and (34) are valid for a wide range of frequencies, they break down as $k_{z} \rightarrow 0$ (i.e., near the linear cutoff frequencies) because the wavenumber shift approaches infinity. However, the basic equations [Eqs. (16) and (22)] governing the temporal and spatial modulations are valid for all frequencies. In this section, we specialize these equations to frequencies near cutoff. Eliminating $\partial^{2} A / \partial T_{1}^{2}$ from Eq. (22) by using Eq. (16), we obtain

$$
2 i\left(\omega \frac{\partial A}{\partial T_{2}}+k_{z} \frac{\partial A}{\partial Z_{2}}\right)+\left(1-\frac{k_{z}^{2}}{\omega^{2}}\right) \frac{\partial^{2} A}{\partial Z_{1}^{2}}=\Lambda A^{2} \bar{A} .
$$

Letting $T_{2}=\delta^{2} t$ and $Z_{n}=\delta^{n} z$ in Eq. (40), we rewrite it as

$$
2 i\left(\omega \frac{\partial A}{\partial t}+k_{z} \frac{\partial A}{\partial z}\right)+\left(1-\frac{k_{z}^{2}}{\omega^{2}}\right) \frac{\partial^{2} A}{\partial z^{2}}=\delta^{2} \Lambda A^{2} \bar{A}
$$

which can also be written in the form

$$
\frac{\partial A}{\partial t}+\omega^{\prime} \frac{\partial A}{\partial z}-\frac{1}{2} i \omega^{\prime \prime} \frac{\partial^{2} A}{\partial z^{2}}=-\frac{1}{2} i \Lambda \omega^{-1} A^{2} \bar{A} .
$$

For monochromatic waves, $\partial A / \partial t=0$ and Eq. (41) becomes 


$$
2 i k_{z} \frac{d A}{d z}+\left(1-\frac{k_{z}^{2}}{\omega^{2}}\right) \frac{d^{2} A}{d z^{2}}=\delta^{2} \Lambda A^{2} \bar{A},
$$

which is valid for all frequencies away from zero. We take the solution of $\mathrm{Eq} .(43)$ in the form

$$
A=\frac{1}{2} a \exp (i \beta),
$$

where $a$ is constant and

$$
\frac{d \beta}{d z}=\left\{-k_{z}+\left[k_{z}^{2}-\frac{1}{4} \delta^{2}\left(1-\frac{k_{z}^{2}}{\omega^{2}}\right) \Lambda a^{2}\right]^{1 / 2}\right\}\left(1-\frac{k_{z}^{2}}{\omega^{2}}\right)^{-1} .
$$

Away from cutoff frequencies, $k_{z}$ is away from zero and the radical in Eq. (45) can be expanded for small $\delta$ yielding,

$$
\frac{d \beta}{d z}=-\frac{1}{8} \delta^{2} \Lambda k_{z}^{-1} a^{2}
$$

in agreement with the monochromatic wave solution obtained above. On the other hand, when $k_{z}-0$, Eq. (45) tends to

$$
\frac{d \beta}{d z}=-k_{z}+\left(k_{z}^{2}-\frac{1}{4} \delta^{2} \Lambda a^{2}\right)^{1 / 2} .
$$

Substituting for $A$ from Eq. (44) into Eq. (11), using Eq. (47), and letting $T_{0}=t$ and $Z_{n}=\delta^{n} z$, we obtain Eq. (33); however, $k$ of Eq. (34) is modified to

$$
k=\left(k_{z}^{2}-\frac{1}{4} \delta^{2} \Lambda a^{2}\right)^{1 / 2} .
$$

Therefore, the cutoff frequencies are solutions of

$$
k_{z}^{2}-\frac{1}{4} \delta^{2} \Lambda a^{2}=0 \text {. }
$$

Since $k_{\varepsilon}^{2}=\omega^{2}-k_{0}^{2}$ according to Eq. (12), then the cutoff frequencies are

$$
\omega=k_{\rho}+\frac{1}{8} \delta^{2} a^{2} \Lambda\left(k_{\rho}\right)+\cdots,
$$

where $\Lambda\left(k_{\rho}\right)$ stands for the value of $\Lambda$ when $\omega=k_{\rho}$. Equation (41) can be used to study the stability of monochromatic solutions as before.

\section{APPENDIX}

$$
\begin{aligned}
F(\rho)= & J_{m}\left(k_{\rho} \rho\right) J_{m}^{\prime 2}\left(k_{\rho} \rho\right)\left\{2 \epsilon_{2} k_{z}^{2}\left[1-\left(k_{z} / k_{\rho}\right)^{2}\right]-\epsilon_{2}\left(\omega k_{z} / k_{\rho}\right)^{2}\right. \\
& \left.+\omega^{4} \mu_{2}\left(\epsilon_{0} / \mu_{\rho}\right)\left[4\left(m / \rho k_{\rho}^{2}\right)^{2}-\left(3 / k_{\rho}^{2}\right)\right]\right\} \\
& +J_{m}^{3}\left(k_{\rho} \rho\right)\left\{2 \epsilon_{2} k_{z}^{2}\left[1+\left(m / \rho k_{\rho}\right)^{2}\right]\left[1+\left(m k_{z} / \rho k_{\rho}^{2}\right)^{2}\right]\right. \\
& -3 \epsilon_{2} \omega^{2}\left[1+\left(m k_{z} / \rho k_{\rho}^{2}\right)^{2}\right]-\omega \mu_{2}\left(\epsilon_{0} / \mu_{0}\right)\left(\omega / k_{\rho}^{2}\right)^{3} \\
& \left.\times(m / \rho)^{2}\left[2(m / \rho)^{2}-k_{\rho}^{2}\right]\right\}+6 \omega \mu_{2} k_{\rho}\left(\epsilon_{0} / \mu_{0}\right)\left(\omega / k_{\rho}\right)^{3} \\
& \times J_{m}^{\prime 2}\left(k_{\rho} \rho\right) J_{m}^{\prime \prime}\left(k_{\rho} \rho\right)-J_{m}^{\prime}\left(k_{\rho} \rho\right) J_{m}^{2}\left(k_{\rho} \rho\right) \\
& \times\left\{\left(2 / \rho^{3}\right)\left(m^{2} / k_{\rho}^{5}\right)\left[\omega^{4} \mu_{2}\left(\epsilon_{0} / \mu_{0}\right)+\epsilon_{2} k_{\varepsilon}^{4}\right]\right\} .
\end{aligned}
$$

*Present address: College of Engineering, Box 800 , Riyadh University, Riyadh, Saudi Arabia.

${ }^{1}$ J. B. Keller and M.H. Millman, Phys. Rev, 181, 1730 (1969).

${ }^{2}$ T. Taniuti and N. Yajima, J. Math. Phys. 10, 1369 (1969). ${ }^{3} \mathrm{~T}$. Taniuti and N. Yajima, J. Math. Phys. 14, 1389 (1973) ${ }^{4}$ A.H. Nayfeh, Perturbation Methods (Wiley-Interscience, New York, 1973), Chap. VI.

${ }^{5}$ A. H. Nayfeh, J. Acoust. Soc. Am. 57, 803 (1975).

${ }^{6}$ S. A. Akhmanov, A. P. Sukhorukov, and R. V. Khokhlov, Usp. Fiz. Nauk. 93, 19.(1967). 